Case Report

\title{
Gastrojejunal Anastomosis Perforation after Gastric Bypass on a Patient with Underlying Pancreatic Cancer: A Case Report and Review of the Literature
}

\author{
Omar Bellorin, ${ }^{1}$ Anna Kundel, ${ }^{2}$ Alexander Ramirez-Valderrama, ${ }^{1}$ and Armando Castro ${ }^{1}$ \\ ${ }^{1}$ Department of General Surgery, New York Hospital Medical Center of Queens/Weill Cornell Medical College, 5645 Main Street, \\ Flushing, NY 11355, USA \\ ${ }^{2}$ Department of Endocrine Surgery, New York University Langone Medical Center, 550 First Avenue, New York, NY 10016, USA \\ Correspondence should be addressed to Omar Bellorin; omarbellorin@gmail.com
}

Received 28 May 2015; Accepted 20 September 2015

Academic Editor: Muthukumaran Rangarajan

Copyright (C) 2015 Omar Bellorin et al. This is an open access article distributed under the Creative Commons Attribution License, which permits unrestricted use, distribution, and reproduction in any medium, provided the original work is properly cited.

\begin{abstract}
Introduction. We describe a case of gastrojejunal anastomosis perforation after gastric bypass on a patient with underlying pancreatic cancer. Case Description. A 54-year-old female with past surgical history of gastric bypass for morbid obesity and recent diagnosis of unresectable pancreatic cancer presents with abdominal pain, peritonitis, and sepsis. Computerized axial tomography scan shows large amount of intraperitoneal free air. The gastric remnant is markedly distended and a large pancreatic head mass is seen. Intraoperative findings were consistent with a perforated ulcer located at the gastrojejunal anastomosis and a distended gastric remnant caused by a pancreatic mass invading and obstructing the second portion of the duodenum. The gastrojejunal perforation was repaired using an omental patch. A gastrostomy for decompression of the remnant was also performed. The patient had a satisfactory postoperative period and was discharged on day 7. Discussion. Perforation of the gastrojejunal anastomosis after Rouxen-Y gastric bypass is an unusual complication. There is no correlation between the perforation and the presence of pancreatic cancer. They represent two different conditions that coexisted. The presence of a gastrojejunal perforation made the surgeon aware of the advanced stage of the pancreatic cancer.
\end{abstract}

\section{Introduction}

We present a case of a patient with history of pancreatic cancer and Roux-en-Y gastric bypass for morbid obesity who presented with acute abdomen secondary to perforation at the gastrojejunum anastomosis (GJA). The ideology, medical and surgical treatment, surveillance, and complications of GJA ulceration are reviewed. The relationship of obesity and cancer and its implications after gastric bypass is also addressed.

\section{Case Description}

54-year-old female with past surgical history of antecolic antegastric Roux-en-Y gastric bypass in 2004 and recently diagnosed with unresectable pancreatic cancer status after chemotherapy presents with severe left upper quadrant abdominal pain and left sided chest pain which began 12 hours prior. Her current BMI is 35 and she still receives treatment for hypertension and diabetes. The pain was sudden in onset, sharp, and radiating to left shoulder. She had no previous episodes and had no recent esophagogastroduodenoscopy (EGD). Physical exam reveals tachycardia, tachypnea, hypotension, and left upper quadrant tenderness and guarding consistent with peritonitis. Computerized axial tomography (CAT) of the abdomen shows moderate to large amount of intraperitoneal free air, likely representing bowel perforation. The gastric remnant is markedly distended and the free air is only seen in the upper abdomen (Figure 1). There is a pancreatic mass obstructing the second portion of the duodenum (Figure 2).

The patient was resuscitated with intravenous fluid and given antibiotics and thereafter was brought to the operating room for exploratory laparotomy.

Intraoperative findings were consistent with a perforated ulcer located at the GJA and a distended gastric remnant 


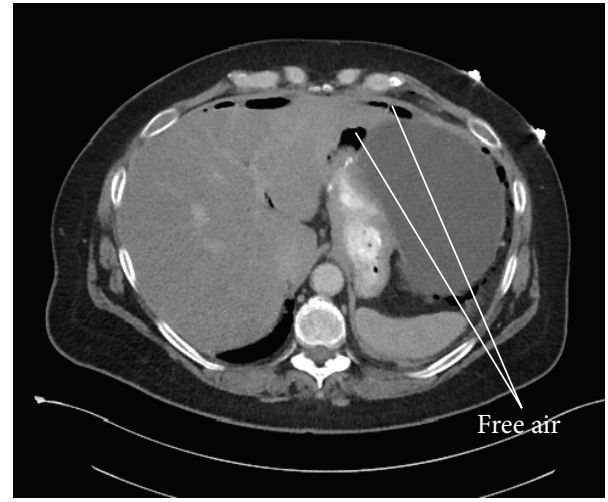

FIGURE 1: CT scan of abdomen showing moderate to large amount of intraperitoneal free air, likely representing bowel perforation.

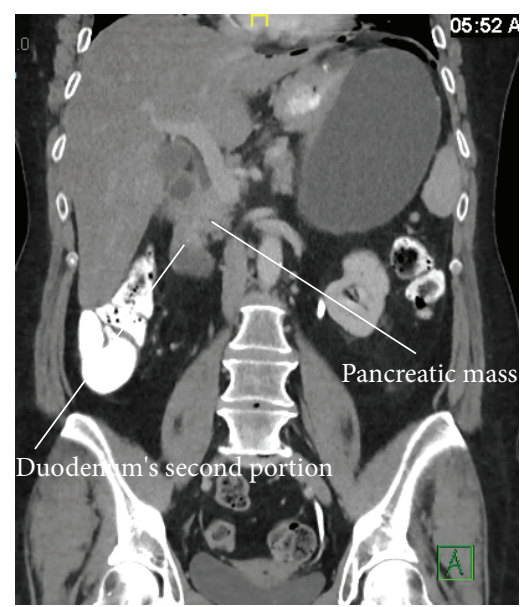

Figure 2: CT scan of abdomen demonstrating a pancreatic mass obstructing the second portion of the duodenum.

caused by a pancreatic mass invading and obstructing the second portion of the duodenum. The abdominal cavity was thoroughly washed and the GJA perforation was repaired using an omental patch (Graham's). A gastrostomy for decompression of the remnant was also performed. The patient had a satisfactory postoperative period and was discharged on day 7.

\section{Discussion}

Patients with gastric bypass may experience a wide variety of complications that can be classified as acute or chronic. Marginal ulcers of the GJA usually represent a chronic complication that the bariatric surgeon encounters frequently.

The reported incidence of marginal ulceration of GJA after gastric bypass varies widely, ranging from 0 to $16 \%$ (Table 1). The risk factors for ulceration are smoking, use of nonsteroidal anti-inflammatory drugs (NSAIDs) and steroids, stress, recent surgery, and the presence of gastrogastric fistulas. Higher incidence has been reported in patients who underwent gastric bypass using circular staplers for the construction of the GJA as opposed to a linear stapler [1-4].
TABLE 1: Reported incidence of marginal ulceration of GJA after gastric bypass.

\begin{tabular}{lc}
\hline Author & Incidence of GJA ulceration (\%) \\
\hline Suggs et al. [1] & 6.3 \\
Higa et al. [12] & 1.4 \\
Gonzalez et al. [2] & 0 \\
Luján et al. [13] & 3.4 \\
DeMaria et al. [14] & 5.1 \\
Kligman et al. [3] & 0.6 \\
Schwartz et al. [4] & 0.8 \\
MacLean et al. [15] & 16 \\
\hline
\end{tabular}

The use of nonabsorbable sutures in the GJA is also associated with marginal ulceration [5]. The presence of Helicobacter pylori may additionally play a role in the development of marginal ulcers. Schirmer et al. [6] described a $2.4 \%$ incidence of marginal ulcers in patients who underwent treatment for Helicobacter pylori preoperatively compared to those who did not (6.8\%).

The majority of these ulcers can be treated medically. However, a subset of patients will have intractable disease requiring surgery for definitive management as the last resort. Patients with marginal ulcers are primarily medically treated with $\mathrm{H} 2$ blockers or proton pump inhibitors. Sucralfate is also added as well as smoking cessation and substitution of ulcerogenic medications. An upper gastrointestinal study is advised if a gastrogastric fistula is suspected and/or patients show no improvement after medical management. There is no consensus on the length of treatment, but the majority of bariatric surgeons choose a minimum of two to three months' regimen followed by an upper endoscopy for confirmation of resolution. Some surgeons advocate the endoscopic removal of nonabsorbable sutures, if present [5].

Clinically the symptoms suggestive of marginal ulceration include, but are not limited to, upper abdominal pain or progressive upper abdominal discomfort and intolerance of food and upper gastrointestinal bleed. Intractability is generally defined as persistence of symptoms after 3 months of medical treatment. Patel et al. [7] reported 39 patients with intractable marginal ulcers whose primary signs and symptoms included chronic abdominal pain (66.6\%), GI bleeding (20.5\%), stomal obstruction (10.2\%), and perforation (2.5\%). A minority of these patients will present with an acute abdomen similar to the patient we presented, and free perforation of the ulcer must be ruled out. Perforation of GJA ulcers is uncommon; the incidence ranges from 0.25 to $1 \%$ [7-10]. The risk factors for perforation are the same as for ulceration; however, smoking, history of recent surgery, and NSAIDs and steroids use are the common denominator in this particular situation [11]. They represent a life-threatening condition with a mortality rate of $10 \%$ [11].

Patients with perforation of a GJA ulcer need aggressive fluid resuscitation and prompt initiation of antibiotic therapy prior to any urgent surgical management. The definitive approach can be performed via open surgery or laparoscopy and consists of primary repair of the ulcer and omental patch 
along with a thorough washout of the abdominal cavity. A gastrostomy for feeding purposes should be performed as well. Large perforations not amenable to primary and patch repair may require revision of the gastrojejunal anastomosis.

The decision to use laparoscopic approach depended solely on the surgeon's expertise and confidence in advanced laparoscopy. There are several studies comparing open versus laparoscopic repair of perforated peptic ulcers that have demonstrated better outcomes in the laparoscopic group [16, 17]. Shorter hospital stay, reduced wound pain, and earlier return to normal activities are the main advantages. Kalaiselvan et al. [11] reported a series of 10 patients presented with perforated GJA ulcers. All patients were treated with abdominal washout, primary closure of the perforation, and omental patch. Five patients were operated on by general surgeons via an open approach and 5 underwent laparoscopic repair by bariatric surgeons. The laparoscopic group experienced lower morbidity, no mortality, and shorter hospital stay compared to those who underwent open surgery.

Postoperatively, these patients should have a reduction of risk factors, prolonged $\mathrm{H} 2$ blockers/proton pump inhibitors regimen, and eradication of Helicobacter pylori on those tested positive.

Also an upper endoscopy 3 months after the procedure to assess the GJA is recommended.

Obesity and cancer are strongly related. In the United States, approximately 85,000 new cases of patients with cancer per year are related to obesity [18]. Studies have found that an increase of body mass index by $5 \mathrm{~kg} / \mathrm{m}^{2}$ is associated with a $10 \%$ higher cancer-related mortality. On the other hand, patients who undergo bariatric surgery have a lower incidence of cancer and a decrease in cancer-related mortality. This is presumably related to weight loss as demonstrated by Adams et al. [19] in a 12.5-year mean follow-up study of patients who underwent gastric bypass surgery compared to severely obese controls.

Early detection and treatment of cancer in patients undergoing bariatric surgery may also play a role. These patients undergo comprehensive gastrointestinal, pulmonary, and cardiovascular workup preoperatively that is not performed routinely in the general population. Moreover, the stomach remnant after gastric bypass will no longer be conveniently accessible, thus making the preoperative assessment of the upper gastrointestinal tract even more important. Zeni et al. [20] reported the presence of Barrett's esophagus in preoperative EGD to be $1.3 \%$, GIST in $0.7 \%$, gastric polyps in $5 \%$, and Helicobacter pylori-associated gastritis and duodenitis in $27 \%$ and $6 \%$, respectively. This extensive workup may result in early cancer diagnosis and is possibly part of the reason why patients undergoing obesity surgery have a lower cancerrelated mortality.

Once a gastric bypass for obesity is performed, access to the gastric remnant and the biliary tree becomes complicated. There is no standard recommendation for a routine assessment of the gastric remnant after gastric bypass. Although technically difficult, double balloon enteroscopy is a feasible way to assess the duodenum and the residual stomach when the patient experiences symptoms that warrants further workup. Ultrasound or CT guided percutaneous gastrostomy and subsequent gastroscopy are another option. Combined laparoscopy-endoscopy can be used as last resort for diagnosis and treatment.

This anatomic exclusion certainly may result in a delayed diagnosis and treatment of a gastric/duodenal/pancreatic/ periampular cancer. A locally advanced pancreatic mass in a patient who has undergone a gastric bypass may result in gastric outlet obstruction of the remaining stomach, which may lead to gastric distention, isquemia, and eventual perforation of the gastric remnant. A decompressive gastrostomy of the remnant is the treatment of choice to avoid this complication.

\section{Conclusion}

Perforation of the GJA after Roux-en-Y gastric bypass is an unusual complication. There is no correlation between the perforation and the presence of pancreatic cancer. They represent two different conditions that coexisted. The presence of a gastrojejunal perforation made the surgeon aware of the advanced stage of the pancreatic cancer that otherwise would have remained undetected for longer time. Overall the approach performed on the presented patient corresponds to the standard and the current literature. The laparoscopic approach by an experienced surgeon may afford the patient the advantages of minimally invasive surgery.

\section{Conflict of Interests}

The authors declare that there is no conflict of interests regarding the publication of this paper.

\section{References}

[1] W. J. Suggs, W. Kouli, M. Lupovici, W. Y. Chau, and R. E. Brolin, "Complications at gastrojejunostomy after laparoscopic Roux-en-Y gastric bypass: comparison between 21- and $25 \mathrm{~mm}$ circular staplers," Surgery for Obesity and Related Diseases, vol. 3, no. 5, pp. 508-514, 2007.

[2] R. Gonzalez, E. Lin, K. R. Venkatesh, S. P. Bowers, and C. D. Smith, "Gastrojejunostomy during laparoscopic gastric bypass," Archives of Surgery, vol. 138, no. 2, pp. 181-184, 2003.

[3] M. D. Kligman, C. Thomas, and J. Saxe, "Effect of the learning curve on the early outcomes of laparoscopic Roux-en-Y gastric bypass," American Surgeon, vol. 69, no. 4, pp. 304-309, 2003.

[4] M. L. Schwartz, R. L. Drew, R. W. Roiger, S. R. Ketover, and M. Chazin-Caldie, "Stenosis of the gastroenterostomy after laparoscopic gastric bypass," Obesity Surgery, vol. 14, no. 4, pp. 484-491, 2004.

[5] B. C. Sacks, S. G. Mattar, F. G. Qureshi et al., "Incidence of marginal ulcers and the use of absorbable anastomotic sutures in laparoscopic Roux-en-Y gastric bypass," Surgery for Obesity and Related Diseases, vol. 2, no. 1, pp. 11-16, 2006.

[6] B. Schirmer, C. Erenoglu, and A. Miller, "Flexible endoscopy in the management of patients undergoing Roux-en-Y gastric bypass," Obesity Surgery, vol. 12, no. 5, pp. 634-638, 2002.

[7] R. A. Patel, R. E. Brolin, and A. Gandhi, "Revisional operations for marginal ulcer after Roux-en-Y gastric bypass," Surgery for Obesity and Related Diseases, vol. 5, no. 3, pp. 317-322, 2009. 
[8] M. Lublin, M. McCoy, and D. J. Waldrep, "Perforating marginal ulcers after laparoscopic gastric bypass," Surgical Endoscopy and Other Interventional Techniques, vol. 20, no. 1, pp. 51-54, 2006.

[9] A. M. C. Macgregor, N. E. Pickens, and E. K. Thoburn, "Perforated peptic ulcer following gastric bypass for obesity," American Surgeon, vol. 65, no. 3, pp. 222-225, 1999.

[10] E. L. Felix, J. Kettelle, E. Mobley, and D. Swartz, "Perforated marginal ulcers after laparoscopic gastric bypass," Surgical Endoscopy, vol. 22, no. 10, pp. 2128-2132, 2008.

[11] R. Kalaiselvan, G. Exarchos, N. Hamza, and B. J. Ammori, "Incidence of perforated gastrojejunal anastomotic ulcers after laparoscopic gastric bypass for morbid obesity and role of laparoscopy in their management," Surgery for Obesity and Related Diseases, vol. 8, no. 4, pp. 423-428, 2012.

[12] K. D. Higa, K. B. Boone, and T. Ho, "Complications of the laparoscopic Roux-en-Y gastric bypass: 1,040 patients-what have we learned?" Obesity Surgery, vol. 10, no. 6, pp. 509-513, 2000.

[13] J. A. Luján, M. D. Frutos, Q. Hernández, J. R. Cuenca, G. Valero, and P. Parrilla, "Experience with the circular stapler for the gastrojejunostomy in laparoscopic gastric bypass (350 cases)," Obesity Surgery, vol. 15, no. 8, pp. 1096-1102, 2005.

[14] E. J. DeMaria, H. J. Sugerman, J. M. Kellum, J. G. Meador, and L. G. Wolfe, "Results of 281 consecutive total laparoscopic Rouxen-Y gastric bypasses to treat morbid obesity," Annals of Surgery, vol. 235, no. 5, pp. 640-647, 2002.

[15] L. D. MacLean, B. M. Rhode, C. Nohr, S. Katz, and A. P. H. McLean, "Stomal ulcer after gastric bypass," Journal of the American College of Surgeons, vol. 185, no. 1, pp. 1-7, 1997.

[16] W. T. Siu, H. T. Leong, B. K. B. Law et al., "Laparoscopic repair for perforated peptic ulcer: a randomized controlled trial," Annals of Surgery, vol. 235, no. 3, pp. 313-319, 2002.

[17] V. Minutolo, G. Gagliano, C. Rinzivillo et al., "Laparoscopic surgical treatment of perforated duodenal ulcer," Chirurgia italiana, vol. 61, no. 3, pp. 309-313, 2009.

[18] K. Basen-Engquist and M. Chang, "Obesity and cancer risk: recent review and evidence," Current Oncology Reports, vol. 13, no. 1, pp. 71-76, 2011.

[19] T. D. Adams, A. M. Stroup, R. E. Gress et al., "Cancer incidence and mortality after gastric bypass surgery," Obesity, vol. 17, no. 4, pp. 796-802, 2009.

[20] T. M. Zeni, C. T. Frantzides, C. Mahr et al., "Value of preoperative upper endoscopy in patients undergoing laparoscopic gastric bypass," Obesity Surgery, vol. 16, no. 2, pp. 142-146, 2006. 


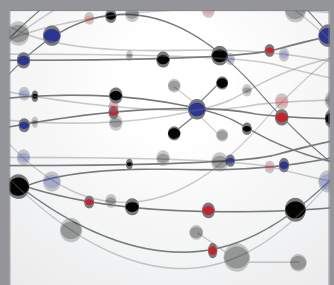

The Scientific World Journal
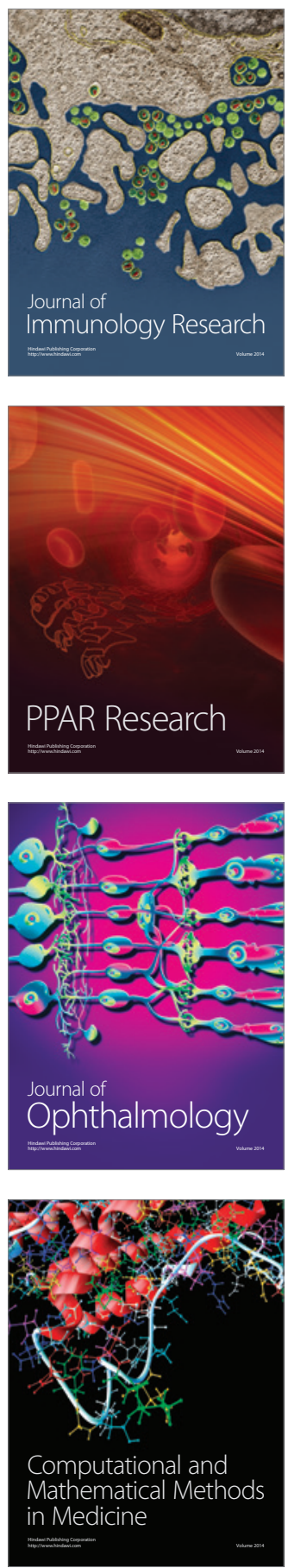

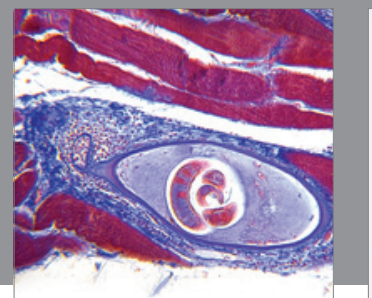

Gastroenterology

Research and Practice
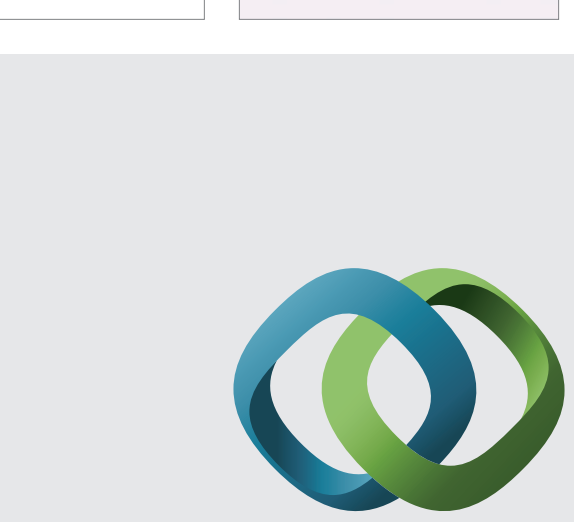

\section{Hindawi}

Submit your manuscripts at

http://www.hindawi.com
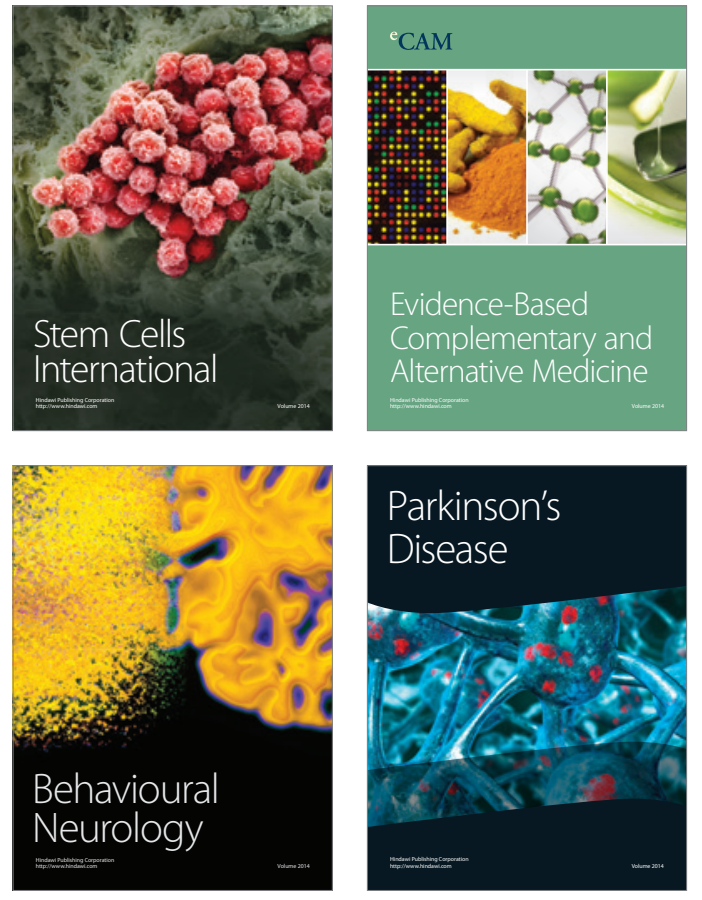
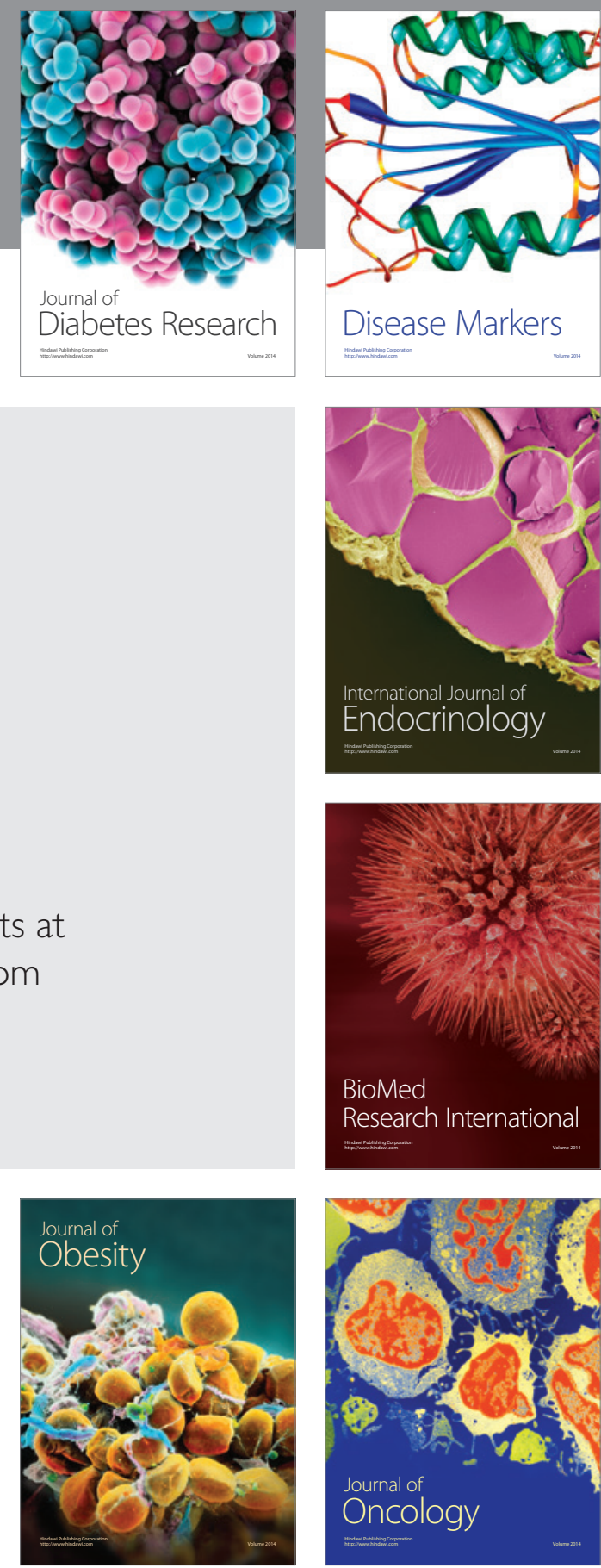

Disease Markers
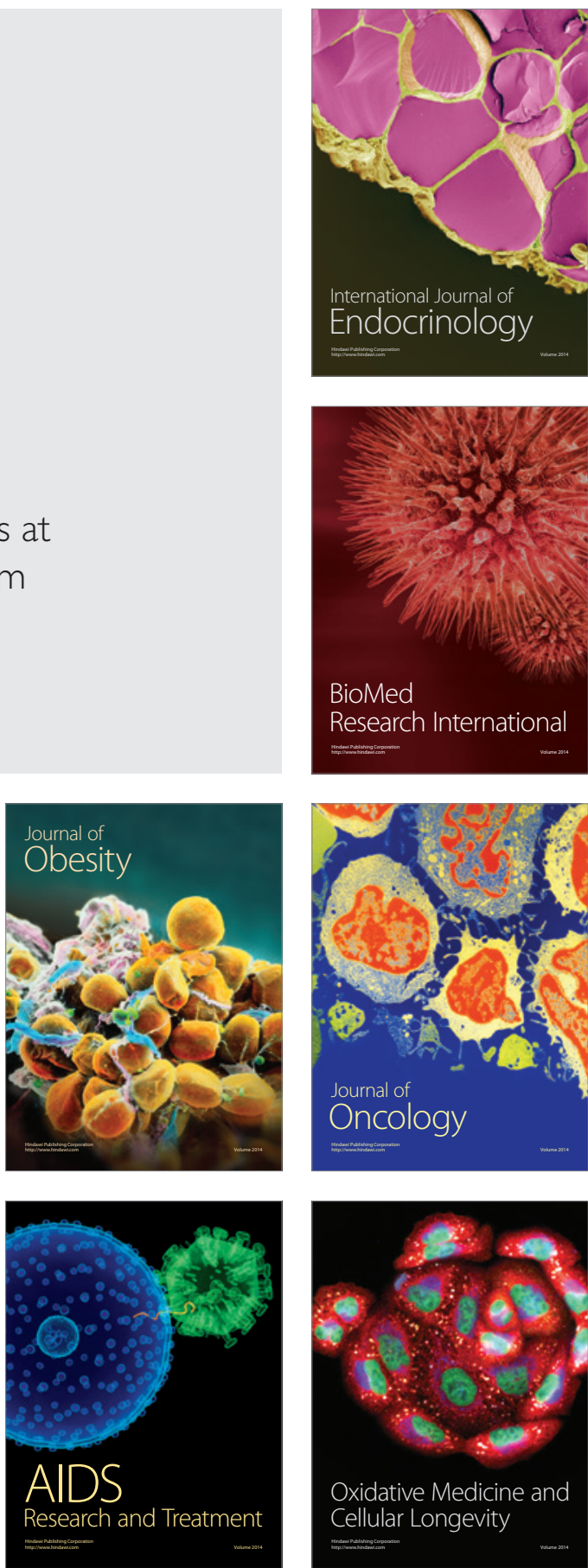